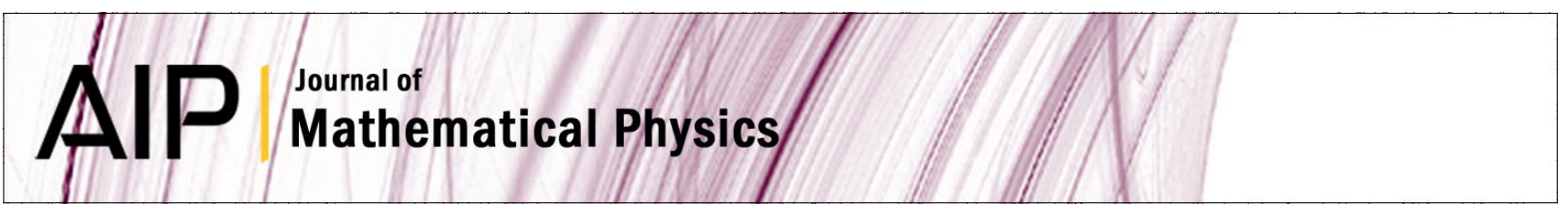

\title{
Poisson bialgebras
}

Xiang $\mathrm{Ni}$ and Chengming Bai

Citation: J. Math. Phys. 54, 023515 (2013); doi: 10.1063/1.4792668

View online: http://dx.doi.org/10.1063/1.4792668

View Table of Contents: http://jmp.aip.org/resource/1/JMAPAQ/v54/i2

Published by the American Institute of Physics.

\section{Related Articles}

Representations of some quantum tori Lie subalgebras

J. Math. Phys. 54, 032302 (2013)

The Poincaré algebra in rank 3 simple Lie algebras

J. Math. Phys. 54, 023508 (2013)

Relativistic Chasles' theorem and the conjugacy classes of the inhomogeneous Lorentz group J. Math. Phys. 54, 022501 (2013)

Localization in abelian Chern-Simons theory

J. Math. Phys. 54, 023507 (2013)

Symmetry classification of variable coefficient cubic-quintic nonlinear Schrödinger equations J. Math. Phys. 54, 023502 (2013)

\section{Additional information on J. Math. Phys.}

Journal Homepage: http://jmp.aip.org/

Journal Information: http://jmp.aip.org/about/about_the_journal

Top downloads: http://jmp.aip.org/features/most_downloaded

Information for Authors: http://jmp.aip.org/authors

\section{ADVERTISEMENT}

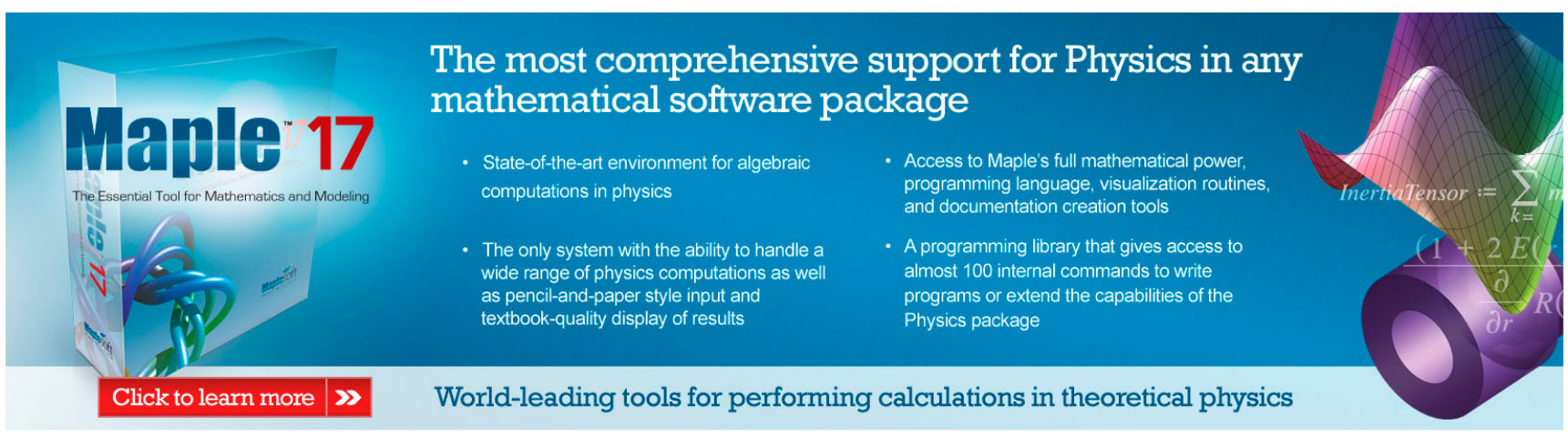




\title{
Poisson bialgebras
}

\author{
Xiang $\mathrm{Ni}^{1}{ }^{1, a)}$ and Chengming $\mathrm{Bai}^{2, \mathrm{~b})}$ \\ ${ }^{1}$ Department of Mathematics, Caltech, Pasadena, California 91125, USA \\ ${ }^{2}$ Chern Institute of Mathematics \& LPMC, Nankai University, Tianjin 300071, People's \\ Republic of China
}

(Received 25 November 2012; accepted 31 January 2013; published online 28 February 2013)

\begin{abstract}
We introduce a notion of Poisson bialgebra as an analogue of a Lie bialgebra of Drinfeld. Poisson bialgebras exhibit many familiar properties of Lie bialgebras. In particular, they can be constructed from a combination of the classical YangBaxter equation and the associative Yang-Baxter equation and there exists a natural Drinfeld classical double. Moreover, Poisson bialgebras are related to certain algebraic structures and they fit naturally into a framework to construct compatible Poisson brackets in integrable systems. @ 2013 American Institute of Physics. [http://dx.doi.org/10.1063/1.4792668]
\end{abstract}

\section{INTRODUCTION}

A Poisson algebra is both a Lie algebra and a commutative associative algebra which are compatible in a certain sense. Poisson algebras play important roles in many fields in mathematics and mathematical physics, such as the Poisson geometry, integrable systems, non-commutative (algebraic or differential) geometry, and so on (Ref. 1 and the references therein).

On the other hand, both Lie algebras and (commutative) associative algebras have a known theory of bialgebras which have been applied to a lot of fields. Lie bialgebras, which were introduced by Drinfeld, ${ }^{2}$ are now well established as the infinitesimalisation of quantum groups. ${ }^{3,4}$ In the case of associative algebras, Joni and Rota introduced the notion of infinitesimal bialgebra in order to provide an algebraic framework for the calculus of divided differences. ${ }^{5}$ With an additional skewsymmetry, it is called an associative D-bialgebra in Ref. 6 (or a balanced infinitesimal bialgebra in Ref. 7 in the opposite algebra, or an antisymmetric infinitesimal bialgebra in Ref. 8). All of these notions are analogues of a Lie bialgebra and Aguiar developed a systematic theory for infinitesimal bialgebras from this point of view. ${ }^{7,9}$ They were also applied in combinatorics. ${ }^{10,11}$

So it is natural to combine the ideas of Lie bialgebras and (commutative and cocommutative) infinitesimal bialgebras in a uniform way, which is the main aim of this paper. More precisely, we establish a bialgebra theory for Poisson algebras, that is, Poisson bialgebras. In fact, a Poisson bialgebra is by definition both a Lie bialgebra (with respect to the Lie bracket of the Poisson algebra) and a commutative and cocommutative infinitesimal bialgebra (with respect to the commutative associative product of the Poisson algebra) which are compatible in a certain sense. Moreover, we will show that a Poisson bialgebra exhibits both the features of a Lie bialgebra and an infinitesimal bialgebra. In particular, a Poisson bialgebra can be obtained by solving the classical Yang-Baxter equation (CYBE) and associative Yang-Baxter equation (AYBE) uniformly (namely, Poisson YangBaxter equation (PYBE)).

A natural application of Poisson bialgebras is that they naturally fit into a framework of Li to construct Poisson brackets in integrable systems. ${ }^{12}$ In fact, Poisson bialgebras can be used to construct the so-called compatible Poisson structures. For example, there naturally exists a family of compatible Poisson structures in the sense of Ref. 12 on the "double space" (Drinfeld classical double) of a finite-dimensional Poisson bialgebra.

\footnotetext{
a)E-mail: xni@caltech.edu.

b) Author to whom correspondence should be addressed. Electronic mail: baicm@nankai.edu.cn.
}

0022-2488/2013/54(2)/023515/14/\$30.00 54, 023515-1 $\quad$ C 2013 American Institute of Physics 
Another important by-product is that there are close relationships between Poisson bialgebras and certain algebraic structures. We introduce notions of $\mathcal{O}$-operator of weight $\lambda \in \mathbb{F}$ ( $\mathbb{F}$ is the base field) of a Poisson algebra as a generalization of the notion of $\mathcal{O}$-operators of a Lie algebra ${ }^{13-15}$ and post-Poisson algebra as a Poisson algebra analogue of a dendriform trialgebra. ${ }^{17}$ We will show that $\mathcal{O}$-operators of weight $\lambda$ can be used to construct post-Poisson algebras and there naturally exists a (non-trivial) post-Poisson algebra structure on the dual space of a Poisson bialgebra arising from a solution of Poisson Yang-Baxter equation.

The paper is organized as follows. In Sec. II, we introduce a notion of matched pair of Poisson algebras which plays an essential role in Sec. III. In Sec. III, we introduce notions of Manin triple of Poisson algebras and Poisson bialgebra and then give the equivalence between them in terms of the matched pairs of Poisson algebras. In Sec. IV, we consider the coboundary cases and get PYBE which is a combination of CYBE and AYBE. In Sec. V, we introduce notions of $\mathcal{O}$-operator of weight $\lambda \in \mathbb{F}$ of a Poisson algebra and post-Poisson algebra and interpret the close relationships between them and Poisson bialgebras. In Sec. VI, we give a natural application of Poisson bialgebras in integrable systems. In Sec. VII, we summarize some conclusions in Secs. II-VI and then make some further discussion.

\section{A. Convention and notations}

In this paper, the base field is taken to be $\mathbb{F}$ unless otherwise specified. This is the field over which we take all the associative, Lie and Poisson algebras, vector spaces, linear maps and tensor products, etc. and the characteristic of $\mathbb{F}$ is zero. All algebras, vector spaces, and manifolds are assumed to be finite-dimensional, although many results still hold in infinite-dimensional case.

Let $(A, \diamond)$ be a vector space with a bilinear operation $\diamond: A \otimes A \rightarrow A$.

(a) Let $L_{\diamond}(x)$ and $R_{\diamond}(x)$ denote the left and right multiplication operator respectively, that is, $L_{\diamond}(x) y=R_{\diamond}(y) x=x \diamond y$ for any $x, y \in A$. We also simply denote them by $L(x)$ and $R(x)$ respectively without confusion. In particular, if $(\mathfrak{g},[]$,$) is a Lie algebra, we let \operatorname{ad}_{[,]}(x)=\operatorname{ad}(x)$ denote the adjoint operator, that is, $\operatorname{ad}_{[,]}(x) y=\operatorname{ad}(x) y=[x, y]$ for any $x, y \in \mathfrak{g}$.

(b) Let $r=\sum_{i} a_{i} \otimes b_{i} \in A \otimes A$. Set

$$
r_{12}=\sum_{i} a_{i} \otimes b_{i} \otimes 1, r_{13}=\sum_{i} a_{i} \otimes 1 \otimes b_{i}, r_{23}=\sum_{i} 1 \otimes a_{i} \otimes b_{i},
$$

where 1 is the unit if $(A, \diamond)$ is unital or a symbol playing a similar role to the unit for the non-unital cases. The operation between two $r$ s is given in an obvious way. For example,

$$
\begin{aligned}
r_{12} \diamond r_{13} & =\sum_{i, j} a_{i} \diamond a_{j} \otimes b_{i} \otimes b_{j}, r_{13} \diamond r_{23}=\sum_{i, j} a_{i} \otimes a_{j} \otimes b_{i} \diamond b_{j}, r_{23} \diamond r_{12} \\
& =\sum_{i, j} a_{j} \otimes a_{i} \diamond b_{j} \otimes b_{i} .
\end{aligned}
$$

(c) Let $V$ be a vector space. Let $\tau: V \otimes V \rightarrow V \otimes V$ be the exchanging operator defined as

$$
\tau(x \otimes y)=y \otimes x, \quad \forall x, y \in V .
$$

(d) Let $V_{1}, V_{2}$ be two vector spaces and $T: V_{1} \rightarrow V_{2}$ be a linear map. Denote the dual (linear) map by $T^{*}: V_{2}^{*} \rightarrow V_{1}^{*}$ defined by

$$
\left\langle v_{1}, T^{*}\left(v_{2}^{*}\right)\right\rangle=\left\langle T\left(v_{1}\right), v_{2}^{*}\right\rangle, \quad \forall v_{1} \in V_{1}, v_{2}^{*} \in V_{2}^{*} .
$$

(e) Let $V$ be a vector space. For any linear map $\rho: A \rightarrow \operatorname{End}_{\mathbb{F}}(V)$, define a linear map $\rho^{*}: A$ $\rightarrow \operatorname{End}_{\mathbb{F}}\left(V^{*}\right)$ by

$$
\left\langle\rho^{*}(x) v^{*}, u\right\rangle=-\left\langle v^{*}, \rho(x) u\right\rangle, \forall x \in A, u \in V, v^{*} \in V^{*} .
$$


Note that in this case, $\rho^{*}$ is different from the one given by Eq. (4) which regards $\operatorname{End}_{\mathbb{F}}(V)$ as a vector space, too.

\section{MODULES AND MATCHED PAIRS OF POISSON ALGEBRAS}

At first, we recall how to construct a Lie algebra (or a commutative associative algebra) structure on the direct sum of two Lie algebras (or two commutative associative algebras) such that both of them are subalgebras.

Let $\left(\mathfrak{g}_{1},[,]_{1}\right)$ and $\left(\mathfrak{g}_{2},[,]_{2}\right)$ be two Lie algebras. If there are $\mathbb{F}$-linear maps $\rho_{1}: \mathfrak{g}_{1} \rightarrow \operatorname{End}_{\mathbb{F}}\left(\mathfrak{g}_{2}\right)$ and $\rho_{2}: \mathfrak{g}_{2} \rightarrow \operatorname{End}_{\mathbb{F}}\left(\mathfrak{g}_{1}\right)$ which are representations of $\mathfrak{g}_{1}$ and $\mathfrak{g}_{2}$, respectively, and they satisfy the following equations:

$$
\begin{aligned}
& \rho_{1}(x)[a, b]_{2}-\left[\rho_{1}(x) a, b\right]_{2}-\left[a, \rho_{1}(x) b\right]_{2}+\rho_{1}\left(\rho_{2}(a) x\right) b-\rho_{1}\left(\rho_{2}(b) x\right) a=0, \\
& \rho_{2}(a)[x, y]_{1}-\left[\rho_{2}(a) x, y\right]_{1}-\left[x, \rho_{2}(a) y\right]_{1}+\rho_{2}\left(\rho_{1}(x) a\right) y-\rho_{2}\left(\rho_{1}(y) a\right) x=0,
\end{aligned}
$$

for any $x, y \in \mathfrak{g}_{1}$ and $a, b \in \mathfrak{g}_{2}$, then $\left(\mathfrak{g}_{1}, \mathfrak{g}_{2}, \rho_{1}, \rho_{2}\right)$ is called a matched pair of Lie algebras. In this case, there exists a Lie algebra structure on the vector space $\mathfrak{g}_{1} \oplus \mathfrak{g}_{2}$ given by

$$
[x+a, y+b]=[x, y]+\rho_{2}(a) y-\rho_{2}(b) x+[a, b]+\rho_{1}(x) b-\rho_{1}(y) a .
$$

It is denoted by $\mathfrak{g}_{1} \triangleright \triangleleft_{\rho_{1}}^{\rho_{2}} \mathfrak{g}_{2}$ or simply $\mathfrak{g}_{1} \triangleright \triangleleft \mathfrak{g}_{2}$. Moreover, every Lie algebra which is the direct sum of the underlying vector spaces of two subalgebras can be obtained from a matched pair of Lie algebras. ${ }^{18,19}$

Let $(A, \circ)$ be a commutative associative algebra. Recall that a representation (or a module) of $A$ is a vector space $V$ and an $\mathbb{F}$-linear map $\mu: A \rightarrow \operatorname{End}_{\mathbb{F}}(V)$ such that $\mu(x \circ y)=\mu(x) \mu(y)$ for any $x$, $y \in A$.

Let $\left(A_{1}, \circ_{1}\right)$ and $\left(A_{2}, \circ_{2}\right)$ be two commutative associative algebras. If there are $\mathbb{F}$-linear maps $\mu_{1}: A_{1} \rightarrow \operatorname{End}_{\mathbb{F}}\left(A_{2}\right)$ and $\mu_{2}: A_{2} \rightarrow \operatorname{End}_{\mathbb{F}}\left(A_{1}\right)$ which are representation of $A_{1}$ and $A_{2}$, respectively, and they satisfy the following equations:

$$
\begin{aligned}
& \mu_{1}(x)\left(a \circ_{2} b\right)=\left(\mu_{1}(x) a\right) \circ_{2} b+\mu_{1}\left(\mu_{2}(a) x\right) b, \\
& \mu_{2}(a)\left(x \circ_{1} y\right)=\left(\mu_{2}(a) x\right) \circ_{1} y+\mu_{2}\left(\mu_{1}(x) a\right) y,
\end{aligned}
$$

for any $x, y \in A_{1}$ and $a, b \in A_{2}$, then $\left(A_{1}, A_{2}, \mu_{1}, \mu_{2}\right)$ is called a matched pair of commutative associative algebras. In this case, there exists a commutative associative algebra structure $\circ$ on the vector space $A_{1} \oplus A_{2}$ given by

$$
(x+a) \circ(y+b)=x \circ_{1} y+\mu_{2}(a) y+\mu_{2}(b) x+a \circ_{2} b+\mu_{1}(x) b+\mu_{1}(y) a .
$$

It is denoted by $A_{1} \bowtie{\mu_{1}}_{\mu_{2}}^{\mu_{2}} A_{2}$ or simply $A_{1} \triangleright \triangleleft A_{2}$. Moreover, every commutative associative algebra which is the direct sum of the underlying vector spaces of two subalgebras can be obtained from a matched pair of commutative associative algebras. ${ }^{8}$

Now we consider the case of Poisson algebras. Recall that a Poisson algebra $(P,\{\},, \circ)$ is an $\mathbb{F}$-vector space equipped with two bilinear operations $\{\},, \circ: P \otimes P \rightarrow P$ such that $(P,\{\}$,$) is a Lie$ algebra and $(P, \circ)$ is a commutative associative algebra and

$$
\{x, y \circ z\}=\{x, y\} \circ z+y \circ\{x, z\}, \quad \forall x, y, z \in P .
$$

A homomorphism between two Poisson algebras is defined as an $\mathbb{F}$-linear map between the two Poisson algebras that preserves the corresponding operations.

Let $(P,\{\},, \circ)$ be a Poisson algebra, $V$ be an $\mathbb{F}$-vector space and $S_{\{,\}}, S_{\circ}: A \rightarrow \operatorname{End}_{\mathbb{F}}(V)$ be two $\mathbb{F}$-linear maps. Then $V$ (or $\left(V, S_{\{,\}}, S_{\circ}\right)$ ) is called a representation (or module) of $P$ if $\left(V, S_{\{,\}}\right)$ is a representation of $(P,\{\}$,$) and \left(V, S_{\circ}\right)$ is a representation of $(P, \circ)$ and they are compatible in the sense that (for any $x, y \in P$ )

$$
S_{\{,\}}(x \circ y)=S_{\circ}(y) S_{\{,\}}(x)+S_{\circ}(x) S_{\{,\}}(y),
$$




$$
S_{\circ}(\{x, y\})=S_{\{,\}}(x) S_{\circ}(y)-S_{\circ}(y) S_{\{,\}}(x) .
$$

In fact, $\left(V, S_{\{,\}}, S_{\circ}\right)$ is a module of a Poisson algebra $(P,\{\},, \circ)$ if and only if the direct sum of vector spaces $P \oplus V$ is turned into a Poisson algebra by defining the operations by (we still denote the operations by $\{$,$\} and \circ$ ):

$$
\begin{aligned}
& \left\{x_{1}+v_{1}, x_{2}+v_{2}\right\}=\left\{x_{1}, x_{2}\right\}+S_{\{,\}}\left(x_{1}\right) v_{2}-S_{\{,\}}\left(x_{2}\right) v_{1}, \\
& \left(x_{1}+v_{1}\right) \circ\left(x_{2}+v_{2}\right)=x_{1} \circ x_{2}+S_{\circ}\left(x_{1}\right) v_{2}+S_{\circ}\left(x_{2}\right) v_{1},
\end{aligned}
$$

for any $x_{1}, x_{2} \in P, v_{1}, v_{2} \in V$. We denote it by $P \ltimes_{S_{\{,\}}, S_{\circ}} V$ or simply $P \ltimes V .{ }^{20}$

Moreover, if $\left(V, S_{\{,\}}, S_{\circ}\right)$ is a module of a Poisson algebra $(P,\{\},, \circ)$, then $\left(V^{*}, S_{\{,\}}^{*},-S_{\circ}^{*}\right)$ is a module of $(P,\{\},, \circ)$, too. Therefore, both $\left(P, \operatorname{ad}_{\{,\}}, L_{\circ}\right)$ and $\left(P^{*}, \operatorname{ad}_{\{,\}}^{*},-L_{\circ}^{*}\right)$ are modules of a Poisson algebra $(P,\{\},, \circ)$.

Theorem 1. Let $\left(P_{1},\{,\}_{1}, \circ_{1}\right)$ and $\left(P_{2},\{,\}_{2}, \circ_{2}\right)$ be two Poisson algebras. Let $\rho_{\{,\}_{1}}, \mu_{\circ_{1}}: P_{1}$ $\rightarrow \operatorname{End}_{\mathbb{F}}\left(P_{2}\right)$ and $\rho_{\{,\}_{2}}, \mu_{\circ_{2}}: P_{2} \rightarrow \operatorname{End}_{\mathbb{F}}\left(P_{1}\right)$ be four $\mathbb{F}$-linear maps such that $\left(P_{1}, P_{2}, \rho_{\{,\}_{1}}, \rho_{\{,\}_{2}}\right)$ is a matched pair of Lie algebras and $\left(P_{1}, P_{2}, \mu_{\circ_{1}}, \mu_{\circ_{2}}\right)$ is a matched pair of commutative associative algebras. If in addition, $\left(P_{2}, \rho_{\{,\}_{1}}, \mu_{\circ_{1}}\right)$ and $\left(P_{1}, \rho_{\{,\}_{2}}, \mu_{\circ_{2}}\right)$ are representations of the Poisson algebras $\left(P_{1},\{,\}_{1}, \circ_{1}\right)$ and $\left(P_{2},\{,\}_{2}, \circ_{2}\right)$ respectively, and $\rho_{\{,\}_{1}}, \rho_{\{,\}_{2}}, \mu_{\circ_{1}}, \mu_{\circ_{2}}$ are compatible in the following sense:

$$
\begin{aligned}
& \rho_{\{,\}_{2}}(a)\left(x \circ_{1} y\right)=\left(\rho_{\{,\}_{2}}(a) x\right) \circ_{1} y+x \circ_{1}\left(\rho_{\{,\}_{2}}(a) y\right)-\mu_{\circ_{2}}\left(\rho_{\{,\}_{1}}(x) a\right) y-\mu_{\circ_{2}}\left(\rho_{\{,\}_{1}}(y) a\right) x, \\
& \left\{x, \mu_{\circ_{2}}(a) y\right\}_{1}-\rho_{\{,\}_{2}}\left(\mu_{\circ_{1}}(y) a\right) x=\mu_{\circ_{2}}\left(\rho_{\{,\}_{1}}(x) a\right) y-\left(\rho_{\{,\}_{2}}(a) x\right) \circ_{1} y+\mu_{\circ_{2}}(a)\left(\{x, y\}_{1}\right), \\
& \rho_{\{,\}_{1}}(x)\left(a \circ_{2} b\right)=\left(\rho_{\{,\}_{1}}(x) a\right) \circ_{2} b+a \circ_{2}\left(\rho_{\{,\}_{1}}(x) b\right)-\mu_{\circ_{1}}\left(\rho_{\{,\}_{2}}(a) x\right) b-\mu_{\circ_{1}}\left(\rho_{\{,\}_{2}}(b) x\right) a, \\
& \left\{a, \mu_{\circ_{1}}(x) b\right\}_{2}-\rho_{\{,\}_{1}}\left(\mu_{\circ_{2}}(b) x\right) a=\mu_{\circ_{1}}\left(\rho_{\{,\}_{2}}(a) x\right) b-\left(\rho_{\{,\}_{1}}(x) a\right) \circ_{2} b+\mu_{\circ_{1}}(x)\left(\{a, b\}_{2}\right),
\end{aligned}
$$

for any $x, y \in P_{1}$ and $a, b \in P_{2}$, then there exists a Poisson algebra structure $\{$,$\} , \circ$ on the vector space $P_{1} \oplus P_{2}$ given by

$$
\begin{gathered}
\{x+a, y+b\}=\left(\{x, y\}_{1}+\rho_{\{,\}_{2}}(a) y-\rho_{\{,\}_{2}}(b) x\right)+\left(\{a, b\}_{2}+\rho_{\{,\}_{1}}(x) b-\rho_{\{,\}_{1}}(y) a\right), \\
(x+a) \circ(y+b)=\left(x \circ_{1} y+\mu_{2}(a) y+\mu_{2}(b) x\right)+\left(a \circ_{2} b+\mu_{1}(x) b+\mu_{1}(y) a\right) .
\end{gathered}
$$

We denote this Poisson algebra by $P_{1} \triangleright \triangleleft_{\rho_{\{,\}_{1}}, \mu_{\circ}, \mu_{2}}^{\rho_{\{\}_{2}}, \mu_{2}} P_{2}$ or simply $P_{1} \bowtie P_{2}$. Moreover, $\left(P_{1}, P_{2}, \rho_{\{,\}_{1}}\right.$, $\left.\mu_{\circ_{1}}, \rho_{\{,\}_{2}}, \mu_{\circ_{2}}\right)$ satisfying the above conditions is called a matched pair of Poisson algebras. On the other hand, every Poisson algebra which is a direct sum of the underlying spaces of two subalgebras can be obtained in the above way.

Proof. In fact, $P_{1} \oplus P_{2}$ becomes a Poisson algebra if and only if the following equations are satisfied (for any $x, y \in P_{1}, a, b \in P_{2}$ ):

$$
\begin{gathered}
\{a,\{x, y\}\}+\{y,\{a, x\}\}+\{x,\{y, a\}\}=0,\{x,\{a, b\}\}+\{b,\{x, a\}\}+\{a,\{b, x\}\}=0, \\
(a \circ x) \circ y=a \circ(x \circ y),(x \circ a) \circ y=x \circ(a \circ y), \quad(x \circ a) \circ b=x \circ(a \circ b),(a \circ x) \circ b \\
=a \circ(x \circ b), \\
\{a, x \circ y\}=\{a, x\} \circ y+x \circ\{a, y\},\{x, a \circ y\}=\{x, a\} \circ y+a \circ\{x, y\}, \\
\{x, a \circ b\}=\{x, a\} \circ b+a \circ\{x, b\},\{a, x \circ b\}=\{a, x\} \circ b+x \circ\{a, b\} .
\end{gathered}
$$

Note that Eq. (23) is equivalent to the fact that $\left(P_{1}, P_{2}, \rho_{\{,\}_{1}}, \rho_{\{,\}_{2}}\right)$ is a matched pair of Lie algebras and Eq. (24) is equivalent to the fact that $\left(P_{1}, P_{2}, \mu_{\circ_{1}}, \mu_{\circ_{2}}\right)$ is a matched pair of commutative 
associative algebras. Moreover, Eqs. (25) and (26) are equivalent to the fact that $\left(\rho_{\{,\}_{1}}, \mu_{\circ_{1}}\right)$ and $\left(\rho_{\{,\}_{2}}, \mu_{\mathrm{O}_{2}}\right)$ satisfy the compatible conditions (13) and (14) and Eqs. (17)-(20).

\section{MANIN TRIPLES OF POISSON ALGEBRAS AND POISSON BIALGEBRAS}

At first, we introduce a notion of Manin triple of Poisson algebras which is an analogue of the notion of Manin triple for Lie algebras. ${ }^{3}$

Definition 1. A Manin triple of Poisson algebras $\left(P, P^{+}, P^{-}\right)$is a triple of Poisson algebras $P$, $P^{+}$, and $P^{-}$together with a nondegenerate symmetric bilinear form $\mathfrak{B}($, ) on $P$ which is invariant in the sense that

$$
\mathfrak{B}(\{x, y\}, z)=\mathfrak{B}(x,\{y, z\}), \quad \mathfrak{B}(x \circ y, z)=\mathfrak{B}(x, y \circ z), \quad \forall x, y, z \in P,
$$

satisfying the following conditions:

(1) $P^{+}$and $P^{-}$are Poisson subalgebras of $P$;

(2) $P=P^{+} \oplus P^{-}$as $\mathbb{F}$-vector spaces;

(3) $P^{+}$and $P^{-}$are isotropic with respect to $\mathfrak{B}($, ).

A homomorphism between two Manin triples of Poisson algebras $\left(P_{1}, P_{1}^{+}, P_{1}^{-}\right)$and $\left(P_{2}, P_{2}^{+}, P_{2}^{-}\right)$with two nondegenerate symmetric invariant bilinear forms $\mathfrak{B}_{1}$ and $\mathfrak{B}_{2}$ respectively is a homomorphism of Poisson algebras $\varphi: P_{1} \rightarrow P_{2}$ such that

$$
\varphi\left(P_{1}^{+}\right) \subset P_{2}^{+}, \quad \varphi\left(P_{1}^{-}\right) \subset P_{2}^{-}, \quad \mathfrak{B}_{1}(x, y)=\varphi^{*} \mathfrak{B}_{2}(x, y)=\mathfrak{B}_{1}(\varphi(x), \varphi(y)), \quad \forall x, y \in P_{1} .
$$

Obviously, a Manin triple of Poisson algebras is just a triple of Poisson algebras such that they are both a Manin triple of Lie algebras ${ }^{3}$ and a commutative associative version of Manin triple ${ }^{8}$ with the same nondegenerate symmetric bilinear form (and share the same isotropic subalgebras). Moreover, it is easy to see that $P^{+}$and $P^{-}$are both Lagrangian subalgebras of $P$.

In particular, there is a special (standard) Manin triple of Poisson algebras as follows. Let $(P,\{\},, \circ)$ be a Poisson algebra. If there is a Poisson algebra structure on the direct sum of the underlying vector space of $P$ and its dual space $P^{*}$ such that $\left(P \oplus P^{*}, P, P^{*}\right)$ is a Manin triple of Poisson algebras with the invariant symmetric bilinear form on $P \oplus P^{*}$ given by

$$
\mathfrak{B}_{p}\left(x+a^{*}, y+b^{*}\right)=\left\langle x, b^{*}\right\rangle+\left\langle a^{*}, y\right\rangle, \quad \forall x, y \in P, a^{*}, b^{*} \in P^{*},
$$

then $\left(P \oplus P^{*}, P, P^{*}\right)$ is called a standard Manin triple of Poisson algebras.

Obviously, a standard Manin triple of Poisson algebras is a Manin triple of Poisson algebras. Conversely, it is easy to show that every Manin triple of Poisson algebras is isomorphic to a standard one. Furthermore, it is straightforward to get the following structure theorem (cf. Refs. 3 and 8).

Structure Theorem (I). Let $\left(P,\{,\}_{1}, \circ_{1}\right)$ and $\left(P^{*},\{,\}_{2}, \circ_{2}\right)$ be two Poisson algebras. Then $\left(P \oplus P^{*}, P, P^{*}\right)$ is a standard Manin triple of Poisson algebras if and only if $\left(P, P^{*}, \operatorname{ad}_{\{,\}_{1}}^{*},-L_{\circ_{1}}^{*}\right.$, $\left.\operatorname{ad}_{\{,\}_{2}}^{*},-L_{\mathrm{O}_{2}}^{*}\right)$ is a matched pair of Poisson algebras.

Like the Manin triples for Lie algebras corresponding to Lie bialgebras ${ }^{3}$ and the commutative associative versions of Manin triples corresponding to commutative and cocommutative infinitesimal bialgebras, ${ }^{6-8,21}$ there is also a bialgebra structure which corresponds to a Manin triple of Poisson algebras following from the equivalent conditions of matched pairs of Poisson algebras. We first recall the notions of Lie bialgebra and infinitesimal bialgebra.

Let $(\mathfrak{g},[]$,$) be a Lie algebra. A Lie bialgebra structure on \mathfrak{g}$ is a skew-symmetric $\mathbb{F}$-linear map $\delta: \mathfrak{g} \rightarrow \mathfrak{g} \otimes \mathfrak{g}$ such that $(\mathfrak{g}, \delta)$ is a Lie coalgebra and $\delta$ is a 1-cocycle of $\mathfrak{g}$ with coefficient in $\mathfrak{g} \otimes \mathfrak{g}$, i.e., it satisfies the following equation:

$$
\delta([x, y])=(\operatorname{ad}(x) \otimes \mathrm{id}+\mathrm{id} \otimes \operatorname{ad}(x)) \delta(y)-(\operatorname{ad}(y) \otimes \mathrm{id}+\mathrm{id} \otimes \operatorname{ad}(y)) \delta(x), \quad \forall x, y \in \mathfrak{g} .
$$


In particular, a Lie bialgebra $(\mathfrak{g}, \delta)$ is called coboundary if $\delta$ is a 1 -coboundary, that is, there exists an $r \in \mathfrak{g} \otimes \mathfrak{g}$ such that

$$
\delta(x)=(\operatorname{ad}(x) \otimes \mathrm{id}+\mathrm{id} \otimes \operatorname{ad}(x)) r, \quad \forall x \in \mathfrak{g} .
$$

In this case, $\delta$ automatically satisfies Eq. (30). It is usually denoted by ( $\mathfrak{g},[], r$,$) .$

Let $(A, \circ)$ be an associative algebra. An infinitesimal bialgebra structure on $A$ is an $\mathbb{F}$-linear map $\Delta: A \rightarrow A \otimes A$ such that $(A, \Delta)$ is a coassociative coalgebra and $\Delta$ is a derivation (1-cocycle), that is,

$$
\Delta(a \circ b)=\left(L_{\circ}(a) \otimes \mathrm{id}\right) \Delta(b)+\left(\mathrm{id} \otimes R_{\circ}(b)\right) \Delta(a), \quad \forall a, b \in A .
$$

In particular, an infinitesimal bialgebra $(A, \Delta)$ is called coboundary if $\Delta$ is a principal derivation (1-coboundary), ${ }^{8,9}$ that is, there exists an $r \in A \otimes A$ such that

$$
\Delta(a)=\left(L_{\circ}(a) \otimes \mathrm{id}-\mathrm{id} \otimes R_{\circ}(a)\right) r, \quad \forall a \in A .
$$

In this case, $\Delta$ automatically satisfies Eq. (32). It is usually denoted by $(A, \circ, r)$.

Definition 2. Let $(P,\{\},, \circ)$ be a Poisson algebra. Suppose that it is equipped with two comultiplications $\delta, \Delta: P \rightarrow P \otimes P$ such that $(P, \delta, \Delta)$ is a Poisson coalgebra, that is, $(P, \delta)$ is a Lie coalgebra and $(P, \Delta)$ is a coassociative coalgebra which is cocommutative and they satisfy the following compatible condition:

$$
(\mathrm{id} \otimes \Delta) \delta(x)=(\delta \otimes \mathrm{id}) \Delta(x)+(\tau \otimes \mathrm{id})(\mathrm{id} \otimes \delta) \Delta(x), \quad \forall x \in P .
$$

If in addition, $(P,\{\},, \delta)$ is a Lie bialgebra and $(P, \circ, \Delta)$ is a commutative and cocommutative infinitesimal bialgebra and $\delta$ and $\Delta$ are compatible in the following sense $(\forall x, y \in P)$ :

$$
\begin{gathered}
\delta(x \circ y)=\left(L_{\circ}(y) \otimes \mathrm{id}\right) \delta(x)+\left(L_{\circ}(x) \otimes \mathrm{id}\right) \delta(y)+\left(\mathrm{id} \otimes \operatorname{ad}_{\{,\}}(x)\right) \Delta(y)+\left(\mathrm{id} \otimes \operatorname{ad}_{\{,\}}(y)\right) \Delta(x), \\
\Delta(\{x, y\})=\left(\operatorname{ad}_{\{,\}}(x) \otimes \mathrm{id}+\mathrm{id} \otimes \operatorname{ad}_{\{,\}}(x)\right) \Delta(y)+\left(L_{\circ}(y) \otimes \mathrm{id}-\mathrm{id} \otimes L_{\circ}(y)\right) \delta(x),
\end{gathered}
$$

then $(P,\{\},, \circ, \delta, \Delta)$ is called a Poisson bialgebra. A homomorphism between two Poisson coalgebras is defined as an $\mathbb{F}$-linear map between the two Poisson coalgebras that preserves the corresponding cooperations. A homomorphism between two Poisson bialgebras is a homomorphism of both Poisson algebras and Poisson coalgebras.

Structure Theorem (II). Let $\left(P,\{,\}_{1}, \circ_{1}\right)$ be a Poisson algebra equipped with two comultiplications $\delta, \Delta: P \rightarrow P \otimes P$. Suppose that $\delta^{*}, \Delta^{*}: P^{*} \otimes P^{*} \subset(P \otimes P)^{*} \rightarrow P^{*}$ induce a Poisson algebra structure on $P^{*}$, where $\delta^{*}$ and $\Delta^{*}$ correspond to the Lie bracket and the product of the commutative associative algebra respectively. Set $\{,\}_{2}=\delta^{*}, \mathrm{o}_{2}=\Delta^{*}$. Then the following conditions are equivalent:

(1) $\left(P,\{,\}_{1}, \circ_{1}, \delta, \Delta\right)$ is a Poisson bialgebra.

(2) $\left(P, P^{*}, \operatorname{ad}_{l,\}_{1}}^{*},-L_{\mathrm{o}_{1}}^{*}, \operatorname{ad}_{\ell, l_{2}}^{*},-L_{\mathrm{o}_{2}}^{*}\right)$ is a matched pair of Poisson algebras.

(3) $\quad\left(P \oplus P^{*}, P, P^{*}\right)$ is a standard Manin triple of Poisson algebras with the bilinear form defined by Eq. (29) and the isotropic subalgebras are $P$ and $P^{*}$.

Proof. We only need to prove that the fact (1) holds if and only if the fact (2) holds. In fact, it is known that $(P,\{\},, \delta)$ is a Lie bialgebra if and only if $\left(P, P^{*}, \operatorname{ad}_{\{,\}_{1}}^{*}, \operatorname{ad}_{\{,\}_{2}}^{*}\right)$ is a matched pair of Lie algebras ${ }^{3}$ and $(P, \circ, \Delta)$ is a commutative and cocommutative infinitesimal bialgebra if and only if $\left(P, P^{*},-L_{\circ_{1}}^{*},-L_{\mathrm{O}_{2}}^{*}\right)$ is a matched pair of commutative associative algebras. ${ }^{8}$ Then by Theorem 1, we only need to prove Eqs. (17)-(20) are equivalent to Eqs. (35) and (36) in the case that $\rho_{\{,\}_{i}}=\operatorname{ad}_{\{,\}_{i}}^{*}, \mu_{\circ_{i}}=-L_{\circ_{i}}^{*}(i=1,2)$. As an example, we give an explicit proof of the case that (for any $x, y \in P, a^{*} \in P^{*}$ )

$$
-\operatorname{ad}_{l,\}_{2}}^{*}\left(a^{*}\right)\left(x \circ_{1} y\right)+\left(\operatorname{ad}_{l,\}_{2}}^{*}\left(a^{*}\right) x\right) \circ_{1} y+L_{\circ_{2}}^{*}\left(\operatorname{ad}_{\{,\}_{1}}^{*}(x) a^{*}\right) y+x \circ_{1}\left(\operatorname{ad}_{l,\}_{2}}^{*}\left(a^{*}\right) y\right)+L_{\circ_{2}}^{*}\left(\operatorname{ad}_{l,\}_{1}}^{*}(y) a^{*}\right) x=0
$$


is equivalent to Eq. (35). The proof of other cases is similar. In fact, let the left hand side of the above equation acts on an arbitrary element $b^{*} \in P^{*}$, we have

$$
\begin{aligned}
& -\left\langle x \circ_{1} y,\left\{b^{*}, a^{*}\right\}_{2}\right\rangle-\left\langle x,\left\{L_{\circ_{1}}^{*}(y)\left(b^{*}\right), a^{*}\right\}_{2}\right\rangle-\left\langle y, b^{*} \circ_{2}\left(\operatorname{ad}_{\{,\}_{1}}^{*}(x) a^{*}\right)\right\rangle \\
& -\left\langle y,\left\{L_{\circ_{1}}^{*}(x) b^{*}, a^{*}\right\}_{2}\right\rangle-\left\langle x,\left(\operatorname{ad}_{\{,\}_{1}}^{*}(y) a^{*}\right) \circ_{2} b^{*}\right\rangle \\
= & \left\langle-\delta\left(x \circ_{1} y\right)+\left(L_{\circ_{1}}(y) \otimes \mathrm{id}\right) \delta(x)+\left(\operatorname{id} \otimes \operatorname{ad}_{\{,\}_{1}}(x)\right) \Delta(y)\right. \\
& \left.+\left(L_{\circ_{1}}(x) \otimes \mathrm{id}\right) \delta(y)+\left(\operatorname{id} \otimes \operatorname{ad}_{\{,\}_{1}}(y)\right) \Delta(x), b^{*} \otimes a^{*}\right\rangle .
\end{aligned}
$$

Then the conclusion follows.

Furthermore, it is easy to show that two Manin triples of Poisson algebras are isomorphic if and only if their corresponding Poisson bialgebras are isomorphic.

\section{COBOUNDARY POISSON BIALGEBRAS}

At first, we recall some important results of the coboundary Lie bialgebras and the coboundary infinitesimal bialgebras. For example, they lead to the famous classical Yang-Baxter equation $(\mathrm{CYBE})^{2,3}$ and Aguiar's associative Yang-Baxter equation (AYBE), ${ }^{9}$ respectively.

Let $\mathfrak{g}$ be a Lie algebra and $r \in \mathfrak{g} \otimes \mathfrak{g}$. The linear map $\delta$ defined by Eq. (31) makes $(A, \delta)$ into a Lie coalgebra if and only if the following conditions are satisfied (for any $x \in \mathfrak{g}$ ):

(1) $\quad(\operatorname{ad}(x) \otimes \mathrm{id}+\mathrm{id} \otimes \operatorname{ad}(x))(r+\tau(r))=0$,

(2) $\quad(\operatorname{ad}(x) \otimes \mathrm{id} \otimes \mathrm{id}+\mathrm{id} \otimes \mathrm{ad}(x) \otimes \mathrm{id}+\mathrm{id} \otimes \mathrm{id} \otimes \operatorname{ad}(x))\left(\left[r_{12}, r_{13}\right]+\left[r_{12}, r_{23}\right]+\left[r_{13}, r_{23}\right]\right)=0$.

In particular, the following equation

$$
\mathbf{C}(r)=\left[r_{12}, r_{13}\right]+\left[r_{12}, r_{23}\right]+\left[r_{13}, r_{23}\right]=0
$$

is called classical Yang-Baxter equation (CYBE). ${ }^{3}$

Let $(A, \circ)$ be a commutative associative algebra and $r \in A \otimes A$. The linear map $\Delta$ defined by Eq. (33) makes $(A, \Delta)$ into a cocommutative and coassociative coalgebra if and only if the following conditions are satisfied (for any $x \in A$ ):

(1) $\quad\left(L_{\circ}(x) \otimes \mathrm{id}-\mathrm{id} \otimes L_{\circ}(x)\right)(r+\tau(r))=0$,

(2) $\quad\left(L_{\circ}(x) \otimes \mathrm{id} \otimes \mathrm{id}-\mathrm{id} \otimes \mathrm{id} \otimes L_{\circ}(x)\right)\left(r_{13} \circ r_{12}-r_{12} \circ r_{23}+r_{23} \circ r_{13}\right)=0$.

In particular, the following equation

$$
\mathbf{A}(r)=r_{13} \circ r_{12}-r_{12} \circ r_{23}+r_{23} \circ r_{13}=0
$$

is called associative Yang-Baxter equation (AYBE) (it was also called AYB in Ref. 7).

Next we introduce the notion of coboundary Poisson bialgebra.

Definition 3. A Poisson bialgebra $(P,\{\},, \circ, \delta, \Delta)$ is called coboundary if $\delta$ and $\Delta$ satisfy (for any $x \in P$ )

$$
\begin{gathered}
\delta(x)=\left(\operatorname{ad}_{\{,\}}(x) \otimes \mathrm{id}+\mathrm{id} \otimes \operatorname{ad}_{\{,\}}(x)\right) r, \\
\Delta(x)=\left(L_{\circ}(x) \otimes \mathrm{id}-\mathrm{id} \otimes L_{\circ}(x)\right) r,
\end{gathered}
$$

for some $r \in P \otimes P$. We sometimes denote it by $(P,\{\},, \circ, r)$.

Obviously, a coboundary Poisson bialgebra $(P,\{\},, \circ, r)$ is equivalent to the fact that both $(P$, $\{\}, r$,$) (as a Lie bialgebra) and (P, \circ, r)$ (as an infinitesimal bialgebra) are coboundary. Further, let $(P,\{\},, \circ, r)$ be a coboundary Poisson bialgebra for some $r \in P \otimes P$, then it is easy to show that $\delta$ and $\Delta$ satisfy Eqs. (35) and (36) automatically.

Now we examine when $(P, \delta, \Delta)$ becomes a Poisson coalgebra, where $\delta$ and $\Delta$ are defined by Eqs. (39) and (40) for some $r \in P \otimes P$, respectively. Let $P$ be an $\mathbb{F}$-vector space equipped with two comultiplications $\delta, \Delta: P \rightarrow P \otimes P$. Then $(P, \delta, \Delta)$ becomes a Poisson coalgebra for $\delta$ corresponding 
to the Lie cobracket and $\Delta$ corresponding to the coproduct of the cocommutative coalgebra if and only if $(P, \delta)$ is a Lie coalgebra and $(P, \Delta)$ is a cocommutative coalgebra and

$$
W(x)=(\mathrm{id} \otimes \Delta) \delta(x)-(\delta \otimes \mathrm{id}) \Delta(x)-(\tau \otimes \mathrm{id})(\mathrm{id} \otimes \delta) \Delta(x)=0, \quad \forall x \in P .
$$

Let $(P,\{\},, \circ)$ be a Poisson algebra. Define two comultiplications $\delta, \Delta: P \rightarrow P \otimes P$ by Eqs. (39) and (40) with $r=\sum_{i} a_{i} \otimes b_{i} \in P \otimes P$, respectively. Then in this case (for any $x \in P$ )

$$
\begin{gathered}
W(x)=-\left(\operatorname{ad}_{\{,\}}(x) \otimes \mathrm{id} \otimes \mathrm{id}\right) \mathbf{A}(r)+\left(\mathrm{id} \otimes L_{\circ}(x) \otimes \mathrm{id}-\mathrm{id} \otimes \mathrm{id} \otimes L_{\circ}(x)\right) \mathbf{C}(r) \\
-\sum_{i}\left[\left(\operatorname{ad}_{\{,\}}\left(a_{i}\right) \otimes \mathrm{id}\right)\left(L_{\circ}(x) \otimes \mathrm{id}-\mathrm{id} \otimes L_{\circ}(x)\right)(r+\tau(r))\right] \otimes b_{i} .
\end{gathered}
$$

In fact, it follows from

$$
\begin{aligned}
W(x)= & \sum_{i, j}\left\{x, a_{j}\right\} \otimes b_{j} \circ a_{i} \otimes b_{i}-\left\{x, a_{j}\right\} \otimes a_{i} \otimes b_{j} \circ b_{i}-\left\{x \circ a_{j}, a_{i}\right\} \otimes b_{i} \otimes b_{j} \\
& +\left\{x \circ b_{j}, a_{i}\right\} \otimes a_{j} \otimes b_{i}+a_{j} \otimes\left\{x, b_{j}\right\} \circ a_{i} \otimes b_{i}-a_{i} \otimes\left\{x \circ a_{j}, b_{i}\right\} \otimes b_{j} \\
& -\left\{b_{j}, a_{i}\right\} \otimes x \circ a_{j} \otimes b_{i}-a_{i} \otimes x \circ a_{j} \otimes\left\{b_{j}, b_{i}\right\}-a_{j} \otimes a_{i} \otimes\left\{x, b_{j}\right\} \circ b_{i}+ \\
& \left\{a_{j}, a_{i}\right\} \otimes b_{i} \otimes x \circ b_{j}+a_{i} \otimes\left\{a_{j}, b_{i}\right\} \otimes x \circ b_{j}+a_{i} \otimes a_{j} \otimes\left\{x \circ b_{j}, b_{i}\right\},
\end{aligned}
$$

which the sum of the first four terms is

$$
-\left(\operatorname{ad}_{\{,\}}(x) \otimes \mathrm{id} \otimes \mathrm{id}\right) \mathbf{A}(r)-\sum_{i}\left[\left(\operatorname{ad}_{\{,\}}\left(a_{i}\right) \otimes 1\right)\left(L_{\circ}(x) \otimes \mathrm{id}\right)(r+\tau(r))\right] \otimes b_{i},
$$

the sum of 5th to 8th is (id $\otimes L_{\circ}(x) \otimes$ id) $\mathbf{C}(r)+\sum_{i}\left[\left(\operatorname{ad}_{\{,\}}\left(a_{i}\right) \otimes L_{\circ}(x)\right)(r+\tau r)\right] \otimes b_{i}$ and the sum of the last four terms is $-\left(\mathrm{id} \otimes \mathrm{id} \otimes L_{\circ}(x)\right) \mathbf{C}(r)$.

Therefore the following conclusion follows:

Theorem 2. Let $(P,\{\},, \circ)$ be a Poisson algebra and $r \in P \otimes P$. Then the comultiplications $\delta$ and $\Delta$ defined by Eqs. (39) and (40), respectively, make $(P, \delta, \Delta)$ into a Poisson coalgebra such that $(P, \circ,\{\},, \delta, \Delta)$ is a Poisson bialgebra if and only if the following conditions are satisfied (for any $x \in P)$ :

(1) $\quad\left(\operatorname{ad}_{\{,\}}(x) \otimes \mathrm{id}+\mathrm{id} \otimes \operatorname{ad}_{\{,\}}(x)\right)(r+\tau(r))=\left(L_{\circ}(x) \otimes \mathrm{id}-\mathrm{id} \otimes L_{\circ}(x)\right)(r+\tau(r))=0 ;$

(2) $\quad\left(L_{\circ}(x) \otimes \mathrm{id} \otimes \mathrm{id}-\mathrm{id} \otimes \mathrm{id} \otimes L_{\circ}(x)\right) \mathbf{A}(r)=0$;

(3) $\quad\left(\operatorname{ad}_{\{,\}}(x) \otimes \mathrm{id} \otimes \mathrm{id}+\mathrm{id} \otimes \operatorname{ad}_{\{,\}}(x) \otimes \mathrm{id}+\mathrm{id} \otimes \mathrm{id} \otimes \operatorname{ad}_{\{,\}}(x)\right) \mathbf{C}(r)=0$;

(4) $W(x)=0$, where $W(x)$ is given by Eq. (42) and $r=\sum_{i} a_{i} \otimes b_{i}$.

Let $(P,\{\},, \circ)$ be a Poisson algebra and $r \in P \otimes P . r$ satisfies Poisson Yang-Baxter equation (PYBE) if $r$ satisfies both CYBE and AYBE. Therefore a direct consequence is that if $r$ is a skewsymmetric solution of PYBE in a Poisson algebra $(P,\{\},, \circ)$, then the comultiplications $\delta$ and $\Delta$ defined by Eqs. (39) and (40), respectively, make $(P, \circ,\{\},, \delta, \Delta)$ into a Poisson bialgebra.

Another important consequence of Theorem 2 is the following Poisson algebra analogue of the Drinfeld double construction.

Theorem 3. Let $\left(P,\{,\}_{1}, \circ_{1}, \delta, \Delta\right)$ be a Poisson bialgebra. Then there is a canonical coboundary Poisson bialgebra structure on $P \oplus P^{*}$.

Proof. Let $r \in P \otimes P^{*} \subset\left(P \oplus P^{*}\right) \otimes\left(P \oplus P^{*}\right)$ correspond to the identity map id: $P \rightarrow P$. Let $\left\{e_{i}, \ldots, e_{n}\right\}$ be a basis of $P$ and $\left\{e_{1}^{*}, \ldots e_{n}^{*}\right\}$ be its dual basis. Then $r=\sum_{i} e_{i} \otimes e_{i}^{*}$. Suppose that the

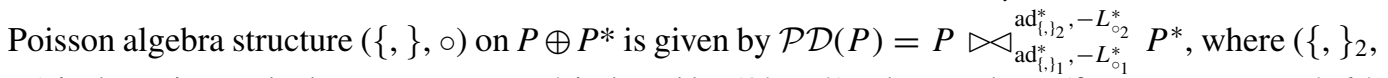
$\left.\mathrm{O}_{2}\right)$ is the Poisson algebra structure on $P^{*}$ induced by $\left(\delta^{*}, \Delta^{*}\right)$. Then we have (for any $x, y \in P, a^{*}, b^{*}$ $\left.\in P^{*}\right)$

$$
\{x, y\}=\{x, y\}_{1}, x \circ y=x \circ_{1} y,\left\{a^{*}, b^{*}\right\}=\left\{a^{*}, b^{*}\right\}_{2}, a^{*} \circ b^{*}=a^{*} \circ_{2} b^{*},
$$




$$
\left\{x, a^{*}\right\}=-\left\{a^{*}, x\right\}=a d_{\{,\}_{1}}^{*}(x) a^{*}-a d_{\{,\}_{2}}^{*}\left(a^{*}\right) x, x \circ a^{*}=a^{*} \circ x=-L_{\circ_{1}}^{*}(x) a^{*}-L_{\circ 2}^{*}\left(a^{*}\right) x .
$$

It is straightforward to prove that $r$ satisfies CYBE and AYBE and

$$
\left(\operatorname{ad}_{\{,\}}(u) \otimes \mathrm{id}+\mathrm{id} \otimes \operatorname{ad}_{\{,\}}(u)\right)(r+\tau(r))=\left(L_{\circ}(u) \otimes \mathrm{id}-L_{\circ}(u) \otimes \mathrm{id}\right)(r+\tau(r))=0, \quad \forall u \in \mathcal{P} \mathcal{D}(P) .
$$

So $r$ satisfies the conditions in Theorem 2. Thus, $\delta_{\mathcal{P D}}(u)=\left(\operatorname{ad}_{\{,\}}(u) \otimes \mathrm{id}+\mathrm{id} \otimes \operatorname{ad}_{\{,\}}(u)\right) r, \quad$ and $\Delta_{\mathcal{P D}}(u)=\left(L_{\circ}(u) \otimes \mathrm{id}-\mathrm{id} \otimes L_{\circ}(u)\right) r, \quad \forall u \in \mathcal{P} \mathcal{D}(P)$, induce a coboundary Poisson bialgebra structure on $\mathcal{P} \mathcal{D}(P)$.

Let $\left(P,\{,\}_{1}, \circ_{1}, \delta_{1}, \Delta_{1}\right)$ be a Poisson bialgebra. With the Poisson-bialgebra structure given in Theorem 3, $P \oplus P^{*}$ is called the Drinfeld classical double of $P$. As in the proof of Theorem 3, we denoted it by $\mathcal{P} \mathcal{D}(P)$.

\section{O-OPERATORS OF POISSON ALGEBRAS, POST-POISSON ALGEBRAS, AND QUASITRIANGULAR POISSON BIALGEBRAS}

In this section we introduce notions of $\mathcal{O}$-operator (of weight $\lambda \in \mathbb{F}$ ) on a Poisson algebra, postPoisson algebra, and quasitriangular Poisson bialgebra. We use $\mathcal{O}$-operators on Poisson algebras to construct post-Poisson algebras. We show that a quasitriangular Poisson bialgebra naturally gives a post-Poisson algebra.

Definition 4. (Refs. 23 and 27). Let $\left(\mathfrak{g},[,]_{\mathfrak{g}}\right)$ and $\left(\mathfrak{k},[,]_{\mathfrak{k}}\right)$ be two Lie algebras. Suppose that $\rho$ is a Lie algebra homomorphism from $\mathfrak{g}$ to $\operatorname{Der}_{\mathbb{F}} \mathfrak{k}$, the Lie algebra consisting of all the derivations of $\mathfrak{k}$. Then $\left(\mathfrak{k},[,]_{\mathfrak{k}}, \rho\right)$ is called a $\mathfrak{g}$-Lie algebra.

Definition 5. (Ref. 24). Let $(A, \circ)$ and $(R, \cdot)$ be two commutative associative algebras. Let $\mu: A \rightarrow \operatorname{End}_{\mathbb{F}}(R)$ be an $\mathbb{F}$-linear map. Then the triple $(R, \cdot, \mu)$ is called an A-module $\mathbb{F}$-algebra if

$$
\mu(x \circ y)=\mu(x) \mu(y), \quad \mu(x)(u \cdot v)=(\mu(x) u) \cdot v, \quad \forall x, y \in A, u, v \in R .
$$

Definition 6. Let $(P,\{\},, \circ)$ and $\left(V,\{,\}_{1}, \circ_{1}\right)$ be two Poisson algebras. Let $S_{\{,\}}, S_{\circ}: P$ $\rightarrow \operatorname{End}_{\mathbb{F}}(R)$ be two $\mathbb{F}$-linear maps such that

(1) $\left(V,\{,\}_{1}, S_{\{,\}}\right)$is a $P$-Lie algebra, where $P$ is seen as a Lie algebra with respect to the Lie bracket $\{$,$\} .$

(2) $\left(V, \circ_{1}, S_{\circ}\right)$ is a $P$-module $\mathbb{F}$-algebra, where $P$ is seen as a commutative associative algebra with respect to the commutative associative product $\circ$.

(3) $\quad\left(V, S_{\{,\}}, S_{\circ}\right)$ is a module of $P$.

(4) The following equations hold:

$$
\begin{gathered}
S_{\{,\}}(x)\left(u \circ_{1} v\right)=\left(S_{\{,\}}(x) u\right) \circ_{1} v+u \circ_{1}\left(S_{\{,\}}(x) v\right), \quad \forall x \in P, u, v \in V, \\
\left\{u, S_{\circ}(x) v\right\}_{1}=-\left(S_{\{,\}}(x) u\right) \circ_{1} v+S_{\circ}(x)\{u, v\}_{1}, \quad \forall x \in P, u, v \in V .
\end{gathered}
$$

Then $\left(V,\{,\}_{1}, \circ_{1}, S_{\{,\}}, S_{\circ}\right)$ is called a P-module Poisson algebra.

In fact, $\left(V,\{,\}_{1}, \bigcirc_{1}, S_{\{,\}}, S_{\circ}\right)$ is a $P$-module Poisson algebra if and only if the direct sum of vector spaces $P \oplus V$ is turned into a Poisson algebra by defining the operations by

$$
\begin{gathered}
\{(x, u),(y, v)\}_{0}=\left(\{x, y\}, S_{\{,\}}(x) v-S_{\{,\}}(y) u+\{u, v\}_{1}\right), \\
(x, u) \circ_{0}(y, v)=\left(x \circ y, S_{\circ}(x) v+S_{\circ}(y) u+u \circ_{1} v\right),
\end{gathered}
$$

for any $x, y \in P, u, v \in V$. Further, if $(P,\{\},, \circ)$ is a Poisson algebra, then it is easy to see that $\left(P,\{\},, \circ, \operatorname{ad}_{\{,\}}, L_{\circ}\right)$ is a $P$-module Poisson algebra. 
Definition 7. (Ref. 25). A (left) Post-Lie algebra is an $\mathbb{F}$-vector space A with two bilinear operations $([],, \diamond)$ satisfying the following equations: (for any $x, y, z \in A$ )

$$
\begin{gathered}
{[x, y]=-[y, x],} \\
{[[x, y], z]+[[z, x], y]+[[y, z], x]=0,} \\
z \diamond(y \diamond x)-y \diamond(z \diamond x)+(y \diamond z) \diamond x-(z \diamond y) \diamond x+[y, z] \diamond x=0, \\
z \diamond[x, y]-[z \diamond x, y]-[x, z \diamond y]=0 .
\end{gathered}
$$

Definition 8. (Ref. 16). A (left) commutative dendriform trialgebra is an $\mathbb{F}$-vector space A equipped with two bilinear operations $(\cdot, \succ)$ satisfying the following equations: (for any $x, y, z \in A$ )

$$
\begin{gathered}
x \cdot y=y \cdot x, \\
(x \cdot y) \cdot z=x \cdot(y \cdot z), \\
(x \succ y+y \succ x+x \cdot y) \succ z=x \succ(y \succ z), \\
(x \succ y) \cdot z=x \succ(y \cdot z) .
\end{gathered}
$$

Definition 9. A (left) post-Poisson algebra is an $\mathbb{F}$-vector space $A$ equipped with four bilinear operations $([],, \diamond, \cdot, \succ)$ such that $(A,[],, \diamond)$ is a (left) post-Lie algebra, $(A, \cdot, \succ)$ is a (left) commutative dendriform trialgebra, and they are compatible in the sense that (for any $x, y, z \in A$ )

$$
\begin{gathered}
{[x, y \cdot z]=[x, y] \cdot z+y \cdot[x, z],} \\
{[x, z \succ y]=z \succ[x, y]-y \cdot(z \diamond x),} \\
x \diamond(y \cdot z)=(x \diamond y) \cdot z+y \cdot(x \diamond z), \\
(y \succ z+z \succ y+y \cdot z) \diamond x=z \succ(y \diamond x)+y \succ(z \diamond x), \\
x \diamond(z \succ y)=z \succ(x \diamond y)+(x \diamond z-z \diamond x+[x, z]) \succ y .
\end{gathered}
$$

Remark 1. (1) Post-Poisson algebra is the Poisson algebra analogue of the dendriform trialgebra of Loday and Ronco. ${ }^{17}$

(2) Let $(A,[],, \diamond, \cdot, \succ)$ be a post-Poisson algebra. If the operations [, ] and $\cdot$ are trivial, then it is a pre-Poisson algebra of Aguiar. ${ }^{26}$

Let $(A,[],, \diamond, \cdot, \succ)$ be a post-Poisson algebra, it is obvious that $(A,[],, \cdot)$ is a Poisson algebra. On the other hand, it is straightforward to get the following conclusion:

Theorem 4. Let $(A,[],, \diamond, \cdot, \succ)$ be a post-Poisson algebra. Define two new bilinear operations $\{\},, \circ: A \otimes A \rightarrow A$ on $A$ by

$$
\{x, y\}=x \diamond y-y \diamond x+[x, y], \quad x \circ y=x \succ y+y \succ x+x \cdot y, \quad \forall x, y \in A .
$$

Then $(A,\{\},, \circ)$ becomes a Poisson algebra. It is called the associated Poisson algebra of $(A$, $[],, \diamond, \cdot, \succ)$ and is denoted by $(P(A),\{\},, \circ)$. Moreover, $\left(P,[],, \cdot, L_{\diamond}, L_{\succ}\right)$ is a P-module Poisson algebra of $(P(A),\{\},, \circ)$. 
The following definition is motivated by the corresponding notions in the context of associative algebras and Lie algebras. ${ }^{24,27}$

Definition 10. Let $(P,\{\},, \circ)$ be a Poisson algebra and $\left(V,\{,\}_{1}, \circ_{1}, S_{\{,\}}, S_{\circ}\right)$ be a $P$-module Poisson algebra. A linear map $\alpha: V \rightarrow P$ is called an $\mathcal{O}$-operator of weight $\lambda \in \mathbb{F}$ associated to $\left(V,\{,\}_{1}, \circ_{1}, S_{\{,\}}, S_{\circ}\right)$ if (for any $u, v \in V$ )

$$
\begin{gathered}
\{\alpha(u), \alpha(v)\}=\alpha\left(S_{\{,\}}(\alpha(u)) v-S_{\{,\}}(\alpha(v)) u+\lambda\{u, v\}_{1}\right), \\
\alpha(u) \circ \alpha(v)=\alpha\left(S_{\circ}(\alpha(u)) v+S_{\circ}(\alpha(v)) u+\lambda u \circ_{1} v\right) .
\end{gathered}
$$

When $\left(V,\{,\}_{1}, \circ_{1}, S_{\{,\}}, S_{\circ}\right)=\left(P,\{\},, \circ, \operatorname{ad}_{\{,\}}, L_{\circ}\right)$, Eqs. (62) and (63) become

$$
\begin{aligned}
& \{\alpha(u), \alpha(v)\}=\alpha(\{\alpha(u), v\}+\{u, \alpha(v)\}+\lambda\{u, v\}), \quad \text { and } \\
& \alpha(u) \circ \alpha(v)=\alpha(\alpha(u) \circ v+u \circ \alpha(v)+\lambda u \circ v),
\end{aligned}
$$

respectively. Equations (64) and (65) imply that $\alpha: P \rightarrow P$ is a Rota-Baxter operator of weight $\lambda \in \mathbb{F}$ on the Lie algebra $(P,\{\}$,$) and on the (commutative) associative algebra (P, \circ)$, respectively. ${ }^{28}$

Theorem 5. Let $(P,\{\},, \circ)$ be a Poisson algebra and $\left(V,\{,\}_{1}, \circ_{1}, S_{\{,\}}, S_{\circ}\right)$ be a P-module Poisson algebra. Let $\alpha: V \rightarrow P$ be an $\mathcal{O}$-operator of weight $\lambda \in \mathbb{F}$ associated to $\left(V,\{,\}_{1}, \circ_{1}, S_{\{,\}}, S_{\circ}\right)$. Define four new bilinear operations $[],, \diamond, \cdot, \succ: V \otimes V \rightarrow V$ on $V$ as follows:

$[u, v]=\lambda\{u, v\}_{1}, \quad u \diamond v=S_{\{,\}}(\alpha(u)) v, \quad u \cdot v=\lambda u \circ_{1} v, \quad u \succ v=S_{\circ}(\alpha(u)) v, \quad \forall u, v \in V$.

Then $(V,[],, \diamond, \cdot, \succ)$ becomes a post-Poisson algebra and $\alpha$ is a homomorphism of Poisson algebras from the associated Poisson algebra $P(V)$ of $(V,[],, \diamond, \cdot, \succ)$ to $(P,\{\},, \circ)$.

Proof: Straightforward.

Definition 11: A coboundary Poisson bialgebra $(P,\{\},, \circ, r)$ is called quasitriangular if $r$ is a solution of PYBE, that is, it satisfies both AYBE and CYBE.

Remark 2. (1) Obviously, a coboundary Poisson bialgebra $(P,\{\},, \circ, r)$ is quasitriangular if and only if $(P,\{\}, r$,$) as a coboundary Lie bialgebra and (P, \circ, r)$ as a coboundary infinitesimal bialgebra are both quasitriangular., 3

(2) If $(P,\{\},, \circ, r)$ is a quasitriangular Poisson bialgebra. Then by Theorem $2, r$ also satisfies the following equations:

$$
\left(\operatorname{ad}_{\{,\}}(x) \otimes \mathrm{id}+\mathrm{id} \otimes \operatorname{ad}_{\{,\}}(x)\right)(r+\tau(r))=\left(L_{\circ}(x) \otimes \mathrm{id}-\mathrm{id} \otimes L_{\circ}(x)\right)(r+\tau(r))=0, \quad \forall x \in P .
$$

Let $V$ be a finite-dimensional $\mathbb{F}$-vector space and let $r \in V \otimes V$. Then $r$ can be identified as a linear map from $V^{*}$ to $V$ which we still denote by $r$ through

$$
\left\langle b^{*}, r\left(a^{*}\right)\right\rangle=\left\langle a^{*} \otimes b^{*}, r\right\rangle, \quad \forall a^{*}, b^{*} \in V^{*} .
$$

Define a linear map $r^{t}: V^{*} \rightarrow V$ by

$$
\left\langle a^{*}, r^{t}\left(b^{*}\right)\right\rangle=\left\langle r, a^{*} \otimes b^{*}\right\rangle .
$$

Obviously $r$ is symmetric or skew-symmetric if and only if $r=r^{t}$ or $r=-r^{t}$ respectively. Define

$$
\alpha=\alpha_{r}=\left(r-r^{t}\right) / 2, \quad \beta=\beta_{r}=\left(r+r^{t}\right) / 2,
$$


called the skew-symmetric part and the symmetric part of $r$ respectively. Then $r=\alpha+\beta$ and $r^{t}=-\alpha+\beta$. Using the results of Refs. 24 and 27 and by a direct computation, we get the following conclusion:

Theorem 6. Let $(P,\{\},, \circ, r)$ be a quasitriangular Poisson bialgebra. Let the linear map $\beta$ be defined by Eq. (70). Define two new bilinear operations [, ], $: P \otimes P \rightarrow P$ as follows:

$$
\left[a^{*}, b^{*}\right]=-2 \operatorname{ad}_{\{,\}}^{*}\left(\beta\left(a^{*}\right)\right) b^{*}, \quad a^{*} \cdot b^{*}=2 L_{\circ}^{*}\left(\beta\left(a^{*}\right)\right) b^{*}, \quad \forall a^{*}, b^{*} \in P^{*} .
$$

Then $\left(P^{*},[],, \cdot, \operatorname{ad}_{\{,\}}^{*},-L_{\circ}^{*}\right)$ becomes a P-module Poisson algebra of $(P,\{\},, \circ)$. Moreover, $r$ regarded as a linear map from $P^{*}$ to $P$ through $E q$. (68) is an $\mathcal{O}$-operator of weight 1 associated to $\left(P^{*},[],, \cdot, \operatorname{ad}_{\{,\}}^{*},-L_{\circ}^{*}\right)$, that is,

$$
\begin{gathered}
\left\{r\left(a^{*}\right), r\left(b^{*}\right)\right\}=r\left(\operatorname{ad}_{\{,\}}^{*}\left(r\left(a^{*}\right)\right) b^{*}-\operatorname{ad}_{\{,\}}^{*}\left(r\left(b^{*}\right)\right) a^{*}+\left[a^{*}, b^{*}\right]\right), \quad \forall a^{*}, b^{*} \in P^{*}, \\
r\left(a^{*}\right) \circ r\left(b^{*}\right)=r\left(-L_{\circ}^{*}\left(r\left(a^{*}\right)\right) b^{*}-L_{\circ}^{*}\left(r\left(b^{*}\right)\right) a^{*}+a^{*} \cdot b^{*}\right), \quad \forall a^{*}, b^{*} \in P^{*} .
\end{gathered}
$$

The following result establishes a close relation between a post-Poisson algebra and a quasitriangular Poisson bialgebra.

Theorem 7. With the conditions and notations in Theorem 6, define four new bilinear operations $[],, \diamond, \cdot, \succ: P^{*} \otimes P^{*} \rightarrow P^{*}$ on $P^{*}$ as follows:

$$
\begin{aligned}
& {\left[a^{*}, b^{*}\right]=-2 \operatorname{ad}_{\{,\}}^{*}\left(\beta\left(a^{*}\right)\right) b^{*}, \quad a^{*} \diamond b^{*}=\operatorname{ad}_{\{,\}}^{*}\left(r\left(a^{*}\right)\right) b^{*},} \\
& a^{*} \cdot b^{*}=2 L_{\circ}^{*}\left(\beta\left(a^{*}\right)\right) b^{*}, \quad a^{*} \succ b^{*}=-L_{\circ}^{*}\left(r\left(a^{*}\right)\right) b^{*}, \quad \forall a^{*}, b^{*} \in P^{*} .
\end{aligned}
$$

Then $\left(P^{*},[],, \diamond, \cdot, \succ\right)$ becomes a post-Poisson algebra and $r$ is a homomorphism of Poisson algebras from the associated Poisson algebra $P\left(P^{*}\right)$ of $\left(P^{*},[],, \diamond, \cdot, \succ\right)$ to $(P,\{\},, \circ)$.

Proof: It follows from Theorems 5 and 6 directly.

\section{RELATIONS WITH INTEGRABLE SYSTEMS}

In this section the study is over the real field $\mathbb{R}$. Recall that two Poisson brackets on the same manifolds are said to be compatible if their sum is also a Poisson bracket. ${ }^{29,30}$ Compatible Poisson brackets play a key role in integrable systems. In Ref. 12, Li addressed the Hamiltonian formulation of Lax equations in the context of Poisson algebras in contrast with the previous results on quadratic Poisson structures on noncommutative associative algebras.

Let $\mathfrak{g}$ be a Lie algebra. A linear operator $R: \mathfrak{g} \rightarrow \mathfrak{g}$ is called a classical $r$-matrix if the $R$-bracket given by

$$
[x, y]_{R}=\frac{1}{2}([R(x), y]+[x, R(y)]), \quad x, y \in \mathfrak{g}
$$

is a Lie bracket, i.e., it satisfies the Jacobi identity.

Let $(P,[],, \circ)$ be a Poisson algebra equipped with a nondegenerate pairing $(\cdot, \cdot)$ which is ad-invariant in the sense that $([x, y], z)=(x,[y, z])$, for any $x, y, z \in P$. A function defined on $P$ is said to be smooth if there exists a map $d F: P \rightarrow P$ such that

$$
\left.\frac{d}{d t}\right|_{t=0} F\left(L+t L^{\prime}\right)=\left(d F(L), L^{\prime}\right), \quad L, L^{\prime} \in P .
$$

Let $M$ be a smooth manifold, we denote the Schouten bracket on $\wedge *(M)$ by [, $]_{S}$. It is a bilinear map [, ] $S_{S}: \wedge^{*}(M) \times \wedge^{*}(M) \rightarrow \wedge^{*}(M)$ which extends the usual Lie bracket operation on $\Gamma(T M)$ and makes $\wedge^{*}(M)$ into a Lie superalgebra.

The main result in Ref. 12 is given as follows. Let $(P,[],, \circ)$ be a Poisson algebra with a Lie bracket $[$,$] and a nondegenerate ad-invariant pairing (\cdot, \cdot)$ with respect to which the operation of 
multiplication is symmetric, i.e., $(x \circ y, z)=(x, y \circ z)$ for all $x, y, z \in P$. Assume that $R: P \rightarrow P$ is a classical $r$-matrix (with respect to the Lie bracket [, ]).

(1) For each integer $n \geq-1$, the formula

$$
\{F, H\}_{(n)}(L)=\left(L,\left[R\left(L^{n+1} d f(L)\right), d H(L)\right]+\left[d F(L), R\left(L^{n+1} d H(L)\right)\right]\right), \quad L \in P,
$$

(where $F$ and $H$ are smooth functions on $P$ ) defines a Poisson structure on $P$.

(2) The structures $\{,\}_{(n)}$ are compatible with each other.

(3) If $\pi_{n}$ is the bivector field corresponding to $\{,\}_{(n)}$ and $D_{\pi_{(n)}}: \wedge^{*}(P) \rightarrow \wedge^{*}(P)$ is the associated coboundary operator, i.e., $D_{\pi_{n}} X=\left[\pi_{n}, X\right]_{S}, X \in \wedge^{*}(P)$, then there exist vector fields $V_{m}$ on $P, m \geq-1$ satisfying the Virasoro relations $\left[V_{m}, V_{n}\right]=(n-m) V_{m+n}$ such that $D_{\pi_{n}}$ $V_{m}=(n-m) \pi_{m+n}, m, n \geq-1$.

Let us come back to Poisson bialgebras. Let $(P,\{\},, \circ, \delta, \Delta)$ be a Poisson bialgebra. Recall that, as a vector space, the Drinfeld classical double $\mathcal{P} \mathcal{D}(P)$ is just $P \oplus P^{*}$. It is known that

$$
R=\frac{1}{2}\left(p_{P^{*}}-p_{P}\right)
$$

is a classical r-matrix on $\mathcal{P} \mathcal{D}(P)$ (as a Lie algebra), where $p_{P^{*}}$ ( $p_{P}$ respectively) is the projection onto $P^{*}\left(P\right.$ respectively). ${ }^{22}$ Moreover, the natural bilinear form $\mathfrak{B}_{p}$ on $\mathcal{P} \mathcal{D}(P)$ defined by Eq. (29) satisfies Eq. (27). So Eq. (78) defines a family of compatible Poisson structures on $\mathcal{P} \mathcal{D}(P)$, where $R$ is defined by Eq. (79).

\section{SUMMARY AND OUTLOOK}

We introduce a notion of Poisson bialgebra which combines a Lie bialgebra and a commutative (and cocommutative) infinitesimal bialgebra which are compatible in a certain sense. It exhibits many familiar properties of Lie bialgebras. It also fits into a framework to construct compatible Poisson brackets in integrable systems and has close relationships with certain algebraic structures like $\mathcal{O}$-operators of weight $\lambda \in \mathbb{F}$ and post-Poisson algebras.

But it is just a study at the beginning which is far way from being well-constructed. For example, we have not known yet whether there is a natural Poisson bialgebra structure on the tensor product of two Poisson bialgebras, like the tensor product of two Poisson algebras still being a Poisson algebra. Furthermore, we will consider to give a similar theory of bialgebra structures on the pre-Poisson algebras introduced by Aguiar. ${ }^{26}$ All of these bialgebras fit into the framework of the generalized bialgebras given by Loday. ${ }^{31}$ Furthermore, Poisson bialgebras might have relations with some other fields, such as the Poisson-Lie T-duality in string theory, ${ }^{32,33}$ the possible "quantization," ${ }^{34}$ some properties of combinatorics, and so on.

\section{ACKNOWLEDGMENTS}

The first author thanks Professor V. N. Zhelyabin for kindly sending his paper to him. This work was supported in part by the National Natural Science Foundation of China (Grant Nos. 10621101, 10920161, 11271202, and 11221091), NKBRPC (Grant No. 2006CB805905), and SRFDP (Grant Nos. 200800550015 and 20120031110022 ).

\footnotetext{
${ }^{1}$ I. Vaisman, Lectures on the Geometry of Poisson Manifolds, Progress in Mathematics Vol. 118 (Birkhäuser Verlag, Basel, 1994).

${ }^{2}$ V. G. Drinfeld, "Hamiltonian structures on Lie groups, Lie bialgebras and the geometric meaning of the classical YangBaxter equations," Sov. Math. Dokl. 27, 68-71 (1983).

${ }^{3}$ V. Chari and A. Pressley, A Guide to Quantum Groups (Cambridge University Press, Cambridge, 1994).

${ }^{4}$ C. Kassel, Quantum Groups, GTM Vol. 155 (Springer-Verlag, Berlin, 1995).

${ }^{5}$ S. A. Joni and G. C. Rota, "Coalgebras and Bialgebras in Combinatorics," Stud. Appl. Math. 61, 93-139 (1979); Reprinted in Gian-Carto Rota on Combinatorics: Introductory Papers and Commentaries, edited by J. P. S. Kung (Birkhäuser, Boston, 1995).

${ }^{6}$ V. N. Zhelyabin, "Jordan bialgebras and their connections with Lie bialgebras," Algebra Logic 36, 1-15 (1997).

${ }^{7}$ M. Aguiar, "On the associative analog of Lie bialgebras," J. Algebra 244, 492-532 (2001).
} 
${ }^{8}$ C. Bai, "Double constructions of Frobenius algebras, Connes cocycles and their duality," J. Noncommu. Geom. 4, 475-530 (2010).

${ }^{9}$ M. Aguiar, Infinitesimal Hopf algebras in New Trends in Hopf Algebra Theory, Contemporary Mathematics, Vol. 267 (American Mathematical Society, Providence, RI, 2000), pp. 1-29.

${ }^{10}$ M. Aguiar, "Infinitesimal Hopf Algebras and the cd-index of Polytopes," Discrete Comput. Geom. 27, 3-28 (2002).

${ }^{11}$ E. Ehrenborg and M. Readdy, "Coproducts and the cd-index," J. Algebraic Combin. 8, 273-299 (1998).

${ }^{12}$ L.-C. Li, "Classical r-matrices and compatible Poisson structures for Lax equations on Poisson algebras," Commun. Math. Phys. 203, 573-592 (1999).

${ }^{13}$ M. Bordemann, "Generalized Lax pairs, the modified classical Yang-Baxter equation, and affine geometry of Lie groups," Commun. Math. Phys. 135, 201-216 (1990).

${ }^{14}$ B. A. Kuperschmidt, "What a classical $r$-matrix really is," J. Nonlinear Math. Phys. 6, 448-488 (1999).

${ }^{15}$ C. Bai, "A unified algebraic approach to the classical Yang-Baxter equation," J. Phys. A: Math. Theor. 40, 11073-11082 (2007).

${ }^{16}$ J.-L. Loday, "On the algebra of quasi-shuffles,” Manuscr. Math. 123, 79-93 (2007).

${ }^{17}$ J.-L. Loday and M. Ronco, "Trialgebras and families of polytopes," in Homotopy Theory: Relations with Algebraic Geometry, Group Cohomology, and Algebraic K-theory, Comteporary Mathematics Vol. 346 (American Mathematical Society, Providence, RI, 2004), pp. 369-398.

${ }^{18}$ S. Majid, "Matched pairs of Lie groups associated to solutions of the Yang-Baxter equation," Pac. J. Math. 141, 311-332 (1990).

${ }^{19}$ M. Takeuchi, "Matched pairs of groups and bismash products of Hopf algebras," Commun. Algebra 9, 841-882 (1981).

${ }^{20}$ R. Schafer, An Introduction to Nonassociative Algebras (Dover, New York, 1995).

${ }^{21}$ M. Aguiar, "Infinitesimal bialgebras, pre-Lie and dendriform algebras," in Hopf algebras, Lecture notes in Pure and Applied Mathematics Vol. 237 (Marcel Dekker, NY, 2004), pp. 1-33.

${ }^{22}$ M. A. Semonov-Tian-Shansky, "What is a classical R-matrix?," Funct. Anal. Appl. 17, 259-272 (1983).

${ }^{23}$ A. W. Knapp, Lie Groups Beyond an Introduction (Birkhäuser, Berlin, 1996).

${ }^{24} \mathrm{C}$. Bai, L. Guo, and X. Ni, “O-operators on associative algebras and associative Yang-Baxter equations," Pac. J. Math. 256, 257-289 (2012).

${ }^{25}$ B. Vallette, "Homology of generalized partition posets," J. Pure Appl. Algebra 208, 699-725 (2007).

${ }^{26}$ M. Aguiar, "Pre-Poisson algebras," Lett. Math. Phys. 54, 263-277 (2000).

${ }^{27}$ C. Bai, L. Guo, and X. Ni, "Nonabelian generalized Lax pairs, the classical Yang-Baxter equation and PostLie algebras," Commun. Math. Phys. 297, 553-596 (2010).

${ }^{28}$ G.-C. Rota, "Baxter operators, an introduction," in Gian-Carlo Rota on Combinatorics: Introductory Papers and Commentaries, edited by J. P. S. Kung (Birkhäuser, Boston, 1995), pp. 504-512.

${ }^{29}$ I. Gelfand and I. Dorfman, "Hamiltonian operators and algebraic structures related to them," Funct. Anal. Appl. 13, 248-262 (1979).

${ }^{30}$ F. Magri, “A simple model of the integrable Hamiltonian equation,” J. Math. Phys. 19, 1156-1162 (1978).

${ }^{31}$ J.-L. Loday, Generalized Bialgebras and Triples of Operads, Astérisque Vol. 320 (Soc. Mathematique France, Paris, 2008), $\mathrm{x}+116 \mathrm{pp}$

${ }^{32}$ C. Klimčík, "Poisson Lie T-duality,” Nuclear Phys. B, Proc. Suppl. 46, 116-121 (1996).

${ }^{33}$ E. J. Beggs and S. Majid, "Poisson-Lie T-duality for quasitriangular Lie bialgebras," Commun. Math. Phys. 220, 455-488 (2001).

${ }^{34}$ P. Etingof and D. Kazhdan, "Quantization of Lie bialgebras, I," Selecta Math., New Ser. 2, 1-41 (1996). 